\title{
Multiple Spinal Metastases from a Malignant Paraganglioma
}

\author{
Chrystal Calderon, MBBS, MRCS ${ }^{*}$ (D), Alberto Perez Villafuerte ${ }^{2}$, Arianne Lalla, MBBS ${ }^{3}$ and \\ Sandeep Maharajh, MBBS ${ }^{4}$
}

Department of Surgery, Eric Williams Medical Sciences Complex, North Central Regional Health Authority, Trinidad and Tobago

*Corresponding author: Chrystal Calderon, MBBS, MRCS, Department of Surgery, Eric Williams Medical Sciences Complex, North Central Regional Health Authority, Trinidad and Tobago, Tel: 1-(868)-225-4673

\begin{abstract}
Background: A malignant paraganglioma is a rare form of cancer that depicts varying symptomatology on presentation. This case serves to highlight an aggressive form of the disease, with considerable metastasis to the cervical, thoracic, and lumbar spine. This case was reliant on histopathological analysis to confirm the diagnosis.

Case summary: A 34-year-old male of Afro-Caribbean descent presented with a 1-month history of shortness of breath, body pains and lower limb weakness. On investigation for a pulmonary embolus, bilateral subpleural densities and several bony lesions were noted. Further imaging of the whole spine showed extensive metastatic lesions with compressive fractures. Ultrasound guided biopsy was performed and confirmed the diagnosis of a paraganglioma as the primary source. Supportive care ensued during in-hospital admission however, the patient demonstrated rapid deterioration of neurological status and progression of pain during this time. He succumbed to this pathological process within days of admission.

Conclusion: A comprehensive understanding and a high level of clinical suspicion of malignant paragangliomas are critical when faced with spinal metastases of unknown origin. The non-specific nature on presentation and potential for rapid neurological deterioration in this aggressive primary tumor is a challenging surgical undertaking.
\end{abstract}

\section{Keywords}

Malignant, Paraganglioma, Spinal metastases, Multiple, Compression fracture

\section{Introduction}

Metastatic spinal cord compression can be highlighted in approximately $10 \%-20 \%$ of cases of advanced primary cancer [1]. The common primary sources of spinal metastatic dissemination include lung, gastrointestinal, renal, prostate and breast malignancies [1,2]. Paragangliomas are rare tumors, with an estimated incidence of 2-10 cases per million, and are seldomly attributed to multiple spinal metastases [3]. They are typically benign neuroendocrine tumors arising from the autonomic paraganglia in the neck, thorax or abdomen [4]. An estimated $10 \%$ of these tumors will demonstrate malignant potential, with possible spread to the bone, lung and liver $[3,5]$.

The end-result of these multiple metastatic spinal deposits is correlated to significant neurological morbidity, fall in overall quality of life and decreased survival rates. The prognostic outcome of malignant paragangliomas is also unfavorable, with a 5-year mortality of less than $50 \%$ [3]. This case patient faced rapid neurological decline and ultimate death, while attempting to diagnose this atypical primary tumor.

\section{Case History}

A 34-year-old male of Afro-Caribbean decent, presented with a 1-month history of shortness of breath with pains to the chest and back. These symptoms were described as insidious in nature but persistent. Associated symptoms included significant weight loss and progressive weakness in the lower limbs bilaterally. He denied any history of palpitations, excessive sweating, or fevers. He had a significant past medical history of admission to the hospital within the last month. During that hospital stay, he was investigated for a retroperitoneal mass with results still pending.

On examination, the patient was noted to have de-

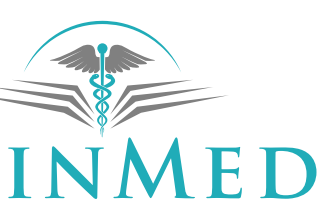

NTERAATIONAL LIBRARY
Citation: Calderon C, Villafuerte AP, Lalla A, Maharajh S (2020) Multiple Spinal Metastases from a Malignant Paraganglioma. Neurosurg Cases Rev 3:041. doi.org/10.23937/2643-4474/1710041

Accepted: September 14, 2020; Published: September 16, 2020

Copyright: (C) 2020 Calderon C, et al. This is an open-access article distributed under the terms of the Creative Commons Attribution License, which permits unrestricted use, distribution, and reproduction in any medium, provided the original author and source are credited. 


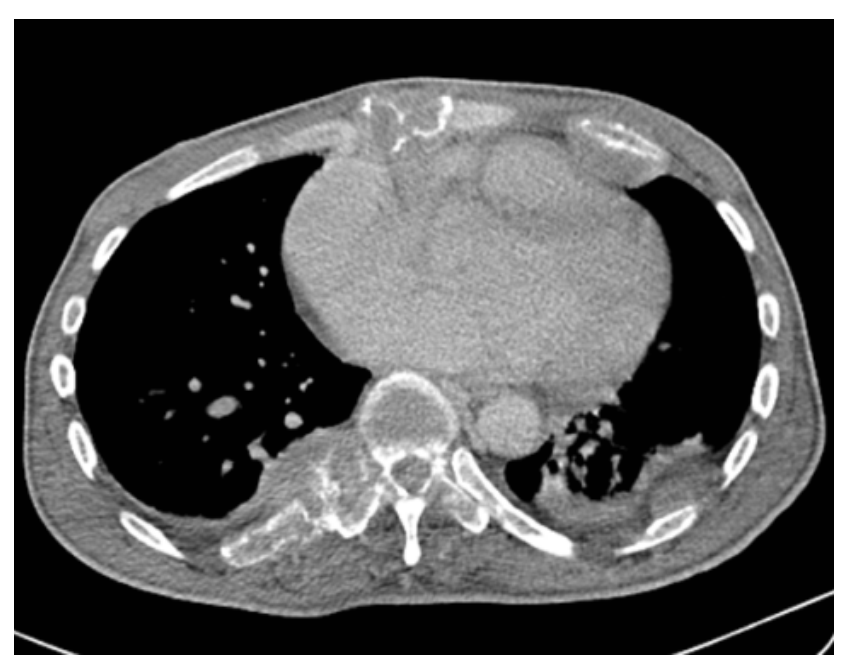

Figure 1: CTPA (axial view) demonstrating subpleural lesions and expansile mass at vertebrocostal joint. creased power in the lower limbs bilaterally-medical research council (MRC) grade 2. Over the next 2 days, the power in his lower limbs declined to MRC grade 0 . Additionally, on pulmonary examination, bilateral decreased air entry was noted on auscultation. A provisional diagnosis of pulmonary embolism was made and a computed tomography pulmonary angiogram (CTPA) was performed. This demonstrated soft tissue subpleural lesions bilaterally with bony erosion and expansion into the T10 vertebra (Figure 1). The case was then referred to the Cardiothoracic and Neurosurgery subspecialties for further management.

Magnetic resonance imaging (MRI) of the whole spinal was conducted. This showed multiple mixed lesions along the vertebrae, with multiple compression fractures throughout the length of the spine. There were also dural based lesions with significant central canal

A.
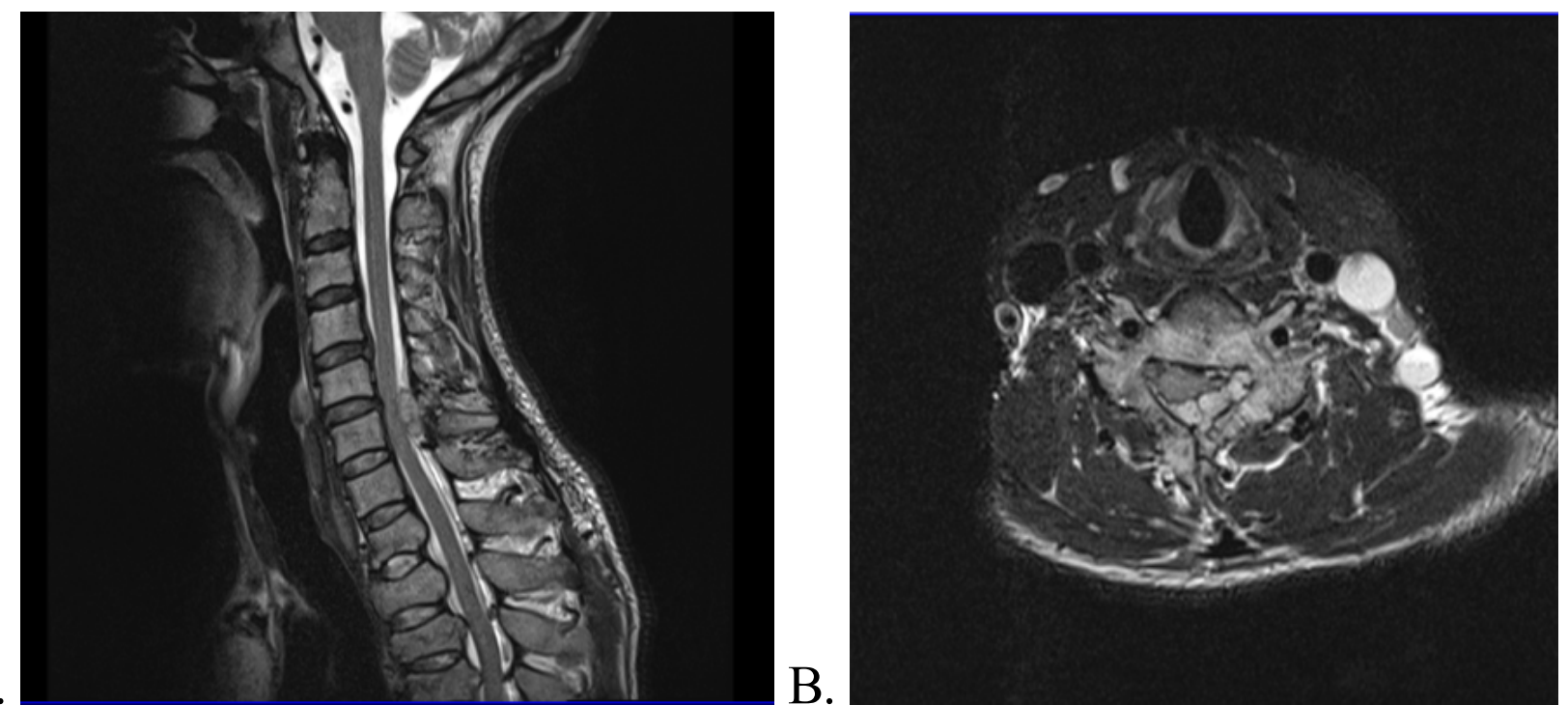

Figure 2: MRI cervical spine (A) Sagittal view; (B) Axial view demonstrating dural based lesion at C6/C7 with spinal canal stenosis.
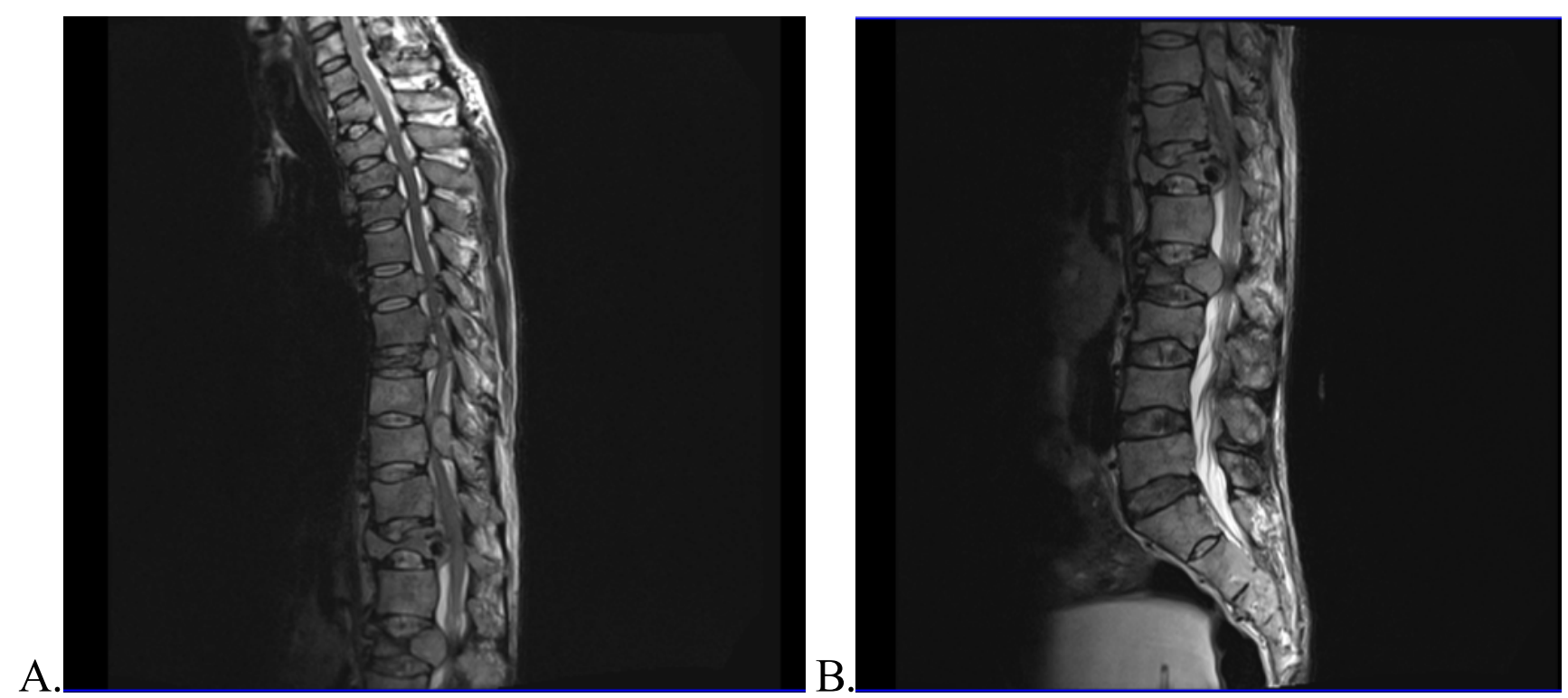

Figure 3: MRI thoraco-lumbar spine T2-weighted images (sagittal views) showing multiple compression fractures (T2, T3, T6, T8, T12, L2), with dural based lesions and corresponding spinal canal stenosis. 
stenosis (Figure 2 and Figure 3). This was in keeping with multiple metastatic spinal lesions of unknown origin. An ultrasound-guided biopsy of the retroperitoneal mass revealed the histopathological diagnosis of a paraganglioma (Figure 4). High dose intravenous dexamethasone for his metastatic disease and low molecular weight heparin for thrombo-prophylaxis was started. The patient and his relatives were thoroughly counselled. Unfortunately, he succumbed to his illness within days of clenching the diagnosis.

\section{Discussion}

Paragangliomas are neuroendocrine tumors which arise from the extra-adrenal autonomic paraganglia. They are closely related to pheochromocytomas of the adrenal gland, as both have originated from embryonic neural crest cells [6]. The combined incidence of pheochromocytomas and paragangliomasis usually estimated as 0.3 per 100,000 of the general population; with the true incidence of paragangliomas not fully known $[7,8]$. Paragangliomas are typically benign, however a less common malignant variant exists. Metastatic spread to the vertebrae is a rare manifestation with limited cases reported in literature. Our case is particularly interesting due to the extensive metastases involving the entire axial spine, further explored in this discussion.

These cases are commonly diagnosed in the third to fifth decades, as seen in this indexed case $[9,10]$. The etiology may be sporadic or hereditary, with $30 \%-40 \%$ of paragangliomas arising from germ-line mutations [10]. The tendency towards malignancy is dependent on various genetic mutations such as succinate dehydrogenase complex and certain syndromes including neurofibromatosis type 1 and von Hippel Lindau syndrome [11,12]. Therefore, genetic testing is advised to be carried out on such patients. Of note, this patient reported no significant family history of undiagnosed or similar tumours, pointing to a likely sporadic origin in this scenario.
Paragangliomas arise from either the sympathetic or parasympathetic ganglia and can demonstrate secretory or non-secretory characteristics [3]. Sympathetic paragangliomas commonly present with episodes of headaches $(80 \%)$, perspiration (71\%), palpitations (64\%), weight loss and hypertension [11,13]. Most parasympathetic paragangliomas are non-functional $(80-90 \%)$ and present as space-occupying lesions with concordant mass effect [8]. Our patient presented with symptoms of shortness of breath with chest and back pains, likely due to his painful metastatic deposits. The coexisting large retroperitoneal mass was an incidental finding on imaging scans from a past hospital visit. Additionally, he denied any catecholamine-related symptoms such as palpitations, excessive sweating, or fever. Therefore, this was highly likely a non-functioning paraganglioma. However, the patient succumbed to the disease before any biochemical tests could have confirmed this.

Biochemical laboratory tests such as the measurement of plasma and urinary metanephrine and catecholamine levels can be done to determine the presence of a secretory paraganglioma. The location of the primary tumor and the presence of metastases can be determined by varied imaging modalities specifically plain radiographs, $\mathrm{CT}, \mathrm{MRI}$ and positron emission tomography scans [3]. Diagnosis is confirmed based on histological assessment of the tumor sample taken at time of biopsy and can only be ascertained after immunohistochemistry analysis. The neuroendocrine nature of the chief cells are confirmed with diffuse, strong positivity for neuron-specific enolase (NSE), synaptophysin, and/or chromogranin, and usually stain negative for keratins [14]. This was demonstrated in this case with a positive synaptophysin, chromogranin, CD56 and reticulin stains, in keeping with a paraganglioma. According to The World Health Organization (WHO), the malignant status of paraganglioma scan only be determined by its metastatic status [15]. Interestingly, histologic features
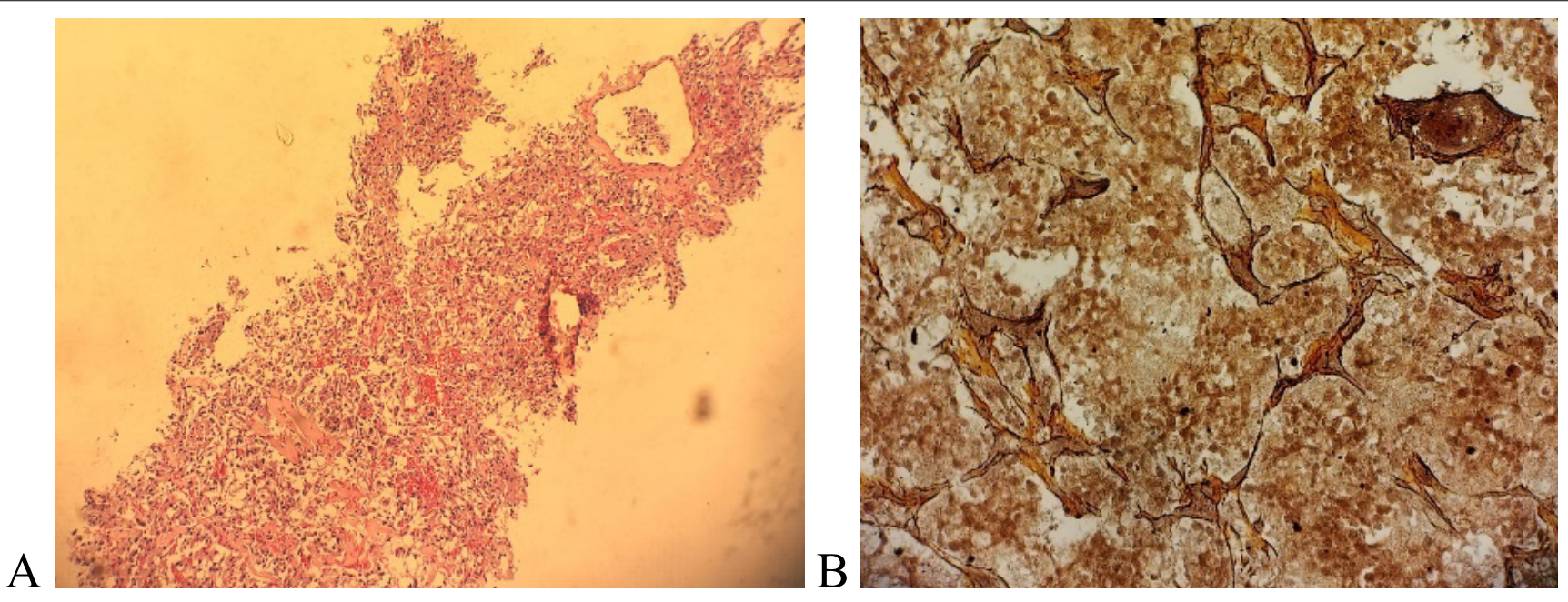

Figure 4: (A) Microscopic (histology) image of specimen showing nests of cells with moderately pleomorphic round nuclei within a prominent vasular network; (B) Immunohistochemical analysis demonstrating postive staining with reticulin (alveolar nesting pattern noted). 
such as nuclear pleomorphism, necrosis, mitotic rate, and local invasion may be seen in benign paragangliomas and are not diagnostic of malignancy [8]. Extra adrenal paragangliomas have greater malignant potential than its counterpart [11]. Our patient was found to have multiple spinal metastases involving cervical, thoracic, and lumbar vertebrae of spinal column, with evidence of considerable neural compromise throughout. Coupling this with the histological findings, a diagnosis of malignant paraganglioma was made.

In the presence of overwhelming metastases, treatment options are severely limited. The main stay of treatment for metastatic disease is mainly palliative. Focus lies on the reduction of tumor burden and the management of catecholamine-related symptoms by surgical resection, chemotherapy and/or radiotherapy [9]. The use of cyclophosphamide, vincristine and dacarbazine (CVD) chemotherapeutic regimen is the widely used in these cases. However, CVD therapy only provides short-term management of metastatic paragangliomas and does not increase overall patient survival [16]. In patients with vertebral metastases and spinal cord compression, it is believed that the treatment of choice is surgical decompression coupled with external beam radiotherapy [5]. Patient prognosis with metastatic paragangliomas is variable. Depending on the study population, the overall 5 -year survival is $35-60 \%$ [11].

\section{Conclusion}

Multiple metastatic spinal disease is linked to pathological vertebral fractures, considerable pain symptoms and severe neurological compromise. Malignant paragangliomas are rarely encountered in clinical practice. More so, with involvement noted in the cervical, thoracic, and lumbar spine with compressive effects. Making the diagnosis of this primary tumor was arduous, especially in the absence of catecholamine-related symptoms, and was solely reliant on a histological diagnosis. A multi-disciplinary team approach is required to provide the best care for these patients, despite the correlated poor prognosis.

\section{Disclosure}

There are no conflicts of interest to be stated. All authors contributed to this manuscript.

\section{References}

1. Wang $\mathrm{F}$, Zhang $\mathrm{H}$, Yang $\mathrm{L}$, Xiong-gang Yang, Hao-ran Zhang, et al. (2019) Epidemiological characteristics of 1196 patients with spinal metastases: A retrospective study. Orthop Surg 11: 1048-1053.
2. Crommett JW (2020) Metastatic spinal cord compression. Oncologic Critical Care.

3. Welander J, Soderkvist P, Gimm O (2018) Genetics and clinical characteristics of hereditary pheochromocytomas and paragangliomas.

4. Quist EE, Javadzadeh BM, Johannesen E, Johansson SL, Lele SM, et al. (2015) Malignant paraganglioma of the bladder: A case report and review of the literature. Pathol Res Pract 211: 183-188.

5. Kapetanakis S, Chourmouzi D, Gkasdaris G, Katsaridis V, Eleftheriadis E, et al. (2018) A rare case of spinal cord compression due to cervical spine metastases from paraganglioma of the jugular foramen-how should it be treated? J Surg Case Reports.

6. Lam AK (2017) Update on adrenal tumours in 2017 world health organization (WHO) of endocrine tumours. Endocr Pathol 28: 213-227.

7. Turchini J, Cheung VKY, Tischler AS, De Krijger RR, Gill AJ (2018) Pathology and genetics of phaeochromocytoma and paraganglioma. Histopathology 72: 97-105.

8. William F Young J, Lynnette K Niema, Kathryn A Mart (2018) Clinical presentation and diagnosis of pheochromocytomas.

9. Liu S, Zhou X, Song A, Zhen Huo, William A Li, et al. (2019) Surgical treatment of malignant paraganglioma with spinal invasion in a juvenile patient: A case report. Medicine (Baltimore) 98: e17145.

10. Costa MHS, Ortiga-Carvalho TM, Violante AD, Vaisman M (2015) Pheochromocytomas and Paragangliomas: Clinical and genetic approaches. Front Endocrinol (Lausanne) 6: 126.

11. Timmers HJLM, Kozupa A, Eisenhofer G (2007) Clinical presentations, biochemical phenotypes, and genotype-phenotype correlations in patients with succinate dehydrogenase subunit b-associated pheochromocytomas and paragangliomas. J Clin Endocrinol Metab 92: 779-786.

12. Shilkrut M, Bar-Deroma R, Bar-Sela G, Berniger A, Kuten A (2010) Low-dose iodine-131 metaiodobenzylguanidine therapy for patients with malignant pheochromocytoma and paraganglioma. Am J Clin Oncol 33: 79-82.

13. Sridhar S, Parghi V (2013) An unusual case of paraganglioma. Indian J Endocrinol Metab 17: 1124-1126.

14. Kliewer KE, Wen D-R, Cancilla PA, Cochran AJ (1989) Paragangliomas: Assessment of prognosis by histologic, immunohistochemical, and ultrastructural techniques. Hum Pathol 20: 29-39.

15. R DeLellis, L Llyod, P UHeitz EC (2004) Pathology and genetics of tumours of endocrine organs. IARC WHO Classification of Tumours, IARC.

16. Nomura K, Kimura H, Shimizu S, Hitomi Kodama, Takahiro Okamoto, et al. (2009) Survival of patients with metastatic malignant pheochromocytoma and efficacy of combined cyclophosphamide, vincristine, and dacarbazine chemotherapy. J Clin Endocrinol Metab 94: 2850-2856.

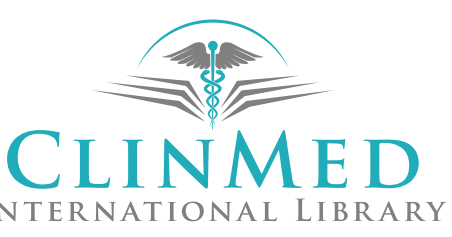

